Overall, this comprehensive reference book concisely describes each complication methodically, with evidencebased information on prevention through to management. It has been designed as a quick reference guide to the diagnosis and management of surgical complications and how to avoid them. It is a useful publication for those involved in the surgical aspects of implantology. However, having previously used Stuart J. Froum's publication Dental implant complications: etiology, prevention, and treatment as a reference text, I feel this is no substitute for the leader in its field.

M. O'Neill

\section{PROSTHETIC REHABILITATION PART II: TECHNICAL PROCEDURES}

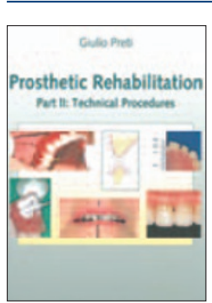

G. Preti

UK: Quintessence

price £165.00; pp 304

ISBN 9781850971986

Prosthetic rehabilitation part II: technical procedures goes well beyond the basic aspects of the technical construction of removable dental prosthesis. The authors have made progressive links from the biological evaluation of individual patients to the influences on the production of removal dentures. There is a strong emphasis on the anatomical significance and the direct impact of this for the development of high quality dental prosthesis. Each of the references in every chapter has been assigned a code indicating the level of evidence. This is particularly good as it allows the reader to quickly and easily identify the quality of the study used.

Each chapter is very concise and explores issues such as soft tissue abnormalities and their relevance to clinical stages of denture production. The book also has a holistic outlook on dental prosthesis, discussing patient attitudes and satisfaction with a view to meeting ever increasing expectations.

The book covers all the main areas of removable dentures. Firstly the basic principles are discussed in detail including retention, stability and support, with the anatomical aspects illustrated by lots of clear step-by-step photos of ideal impressions, border moulding and its relationship to the quality of the appliance. The next aspect covers oral mucosal conditions in those wearing dentures including a chapter on burning mouth syndrome. It explains less frequently discussed issues such as soft tissue conditioners and denture adhesives.

There are further chapters on tooth retained and implant retained overdentures. Finally the book has excellent information regarding oral hygiene and long-term follow up for patients with removal prosthesis.

The nature of this book would appeal both to the generalist and specialist practitioner as it assumes only basic prior knowledge of removable dentures and provides a detailed scientific basis for the technical aspects of prosthetic dentistry, giving practitioners greater confidence in assessing patients and delivering high quality treatment for dentures.

H. Shah

\section{ITI TREATMENT GUIDE, VOLUME 5: SINUS FLOOR ELEVATION PROCEDURES}

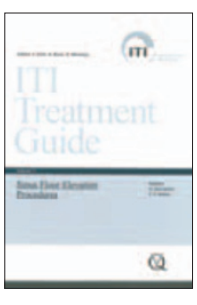

H. Katsuyama, S. S. Jensen

UK: Quintessence

price $€ 68.00$; pp 232

ISBN 9783938947180

Following the success of earlier volumes, the International Team for Implantology (ITI) has produced their latest in the series. This is a comprehensive book exploring the topic of sinus floor elevation procedures. It is aimed at the clinician actively involved in placing implants, although any postgraduate surgeon would benefit from using this reference. The authors and editorial team are made up of leaders in the field of dental implantology.

The recommendations within are derived from the latest ITI consensus conference, 2008, along with the current published evidence. Around half of the total pages are made up from 13 case studies used to illustrate various clinical scenarios. These are well- selected and engaging demonstrations of what the clinician may be faced with when placing an implant in the atrophied maxilla.

The two proposed techniques for maxillary sinus floor elevation - the transcrestal technique and the lateral window technique - are discussed throughout in different sections. The literature on these is reviewed, the surgical techniques described and recommendations on holistic patient treatment put forward. Whilst the finer details of this advanced area of dental implantology are explored, there is no neglect of the fundamentals of patient management. This includes the key anatomy, the relevance of patient history and the radiographical considerations.

One of the great successes of the book is the pleasing illustrations and their consistency. All of the clinical photographs, radiographs, schematic drawings and decision trees offer clarity and understanding. It has fine readability and is edited to the highest standard. With these features in mind, it certainly provides value for money. It meets the needs of the implant surgeon, serving as a valuable resource. However, this area of dentistry is rapidly changing and further research will likely impact on the text's ability to remain current.

I propose this book is the essential text on maxillary sinus floor elevation procedures. Not only an academic reference, it offers a pragmatic and didactic approach to clinical management.

R. A. Scott 